\title{
Concentration Distribution of Solid Particles Transported by a Pseudoplastic Fluid
}

\author{
Aldo Tamburrino', Gonzalo Montserrat ${ }^{2}$, Christian Ihle ${ }^{3}$ \\ ${ }^{1}$ Associate Professor \& Associate Researcher \\ Department of Civil Engineering \& Advanced Mining Technology Center \\ University of Chile \\ Av. Blanco Encalada 2002, Santiago 8370449, Chile \\ atamburr@ing.uchile.cl \\ ${ }^{2}$ Project Engineer \\ Hydraulics National Institute, Minister of Public Works \\ Av. Concordia 0620, Peñaflor 9750913, Chile \\ gonzalomontserrat@inh.cl \\ ${ }^{3}$ Assistant Professor \& Associate Researcher \\ Department of Mining Engineering \& Advanced Mining Technology Center \\ University of Chile \\ Av. Tupper 2069, Santiago, 8370451, Chile \\ cihle@ing.uchile.cl
}

\begin{abstract}
Concentration distributions of solid particles transported by pseudoplastic fluids in laminar regime were determined experimentally. Practically all the distributions show minimum concentration of solids near the centre of the pipe and higher toward the walls and even, for some conditions, the highest values near the top of the pipe. This behaviour of the concentration distribution was explained when diffusive fluxes of the solid particles due to concentration gradient and viscosity gradient are considered. An existing diffusive model was modified in order to take into account negatively buoyant particles and a pseudoplastic fluid as a carrier. It was found that the head loss plays an important role in the fluxes of solid particles due to gradients of concentration and viscosity, increasing them enough to overcome the effect of gravity.
\end{abstract}

Keywords: pseudoplastic fluids, concentration distribution, laminar flow, diffusive fluxes

\section{Introduction and Objective}

The hydraulic transport of solids in pipes is used in many industrial processes, particularly in the mining industry. Usually, it is considered that the flow has to be turbulent to keep the solid particles in suspension, and the upward velocity of the turbulent eddies must be greater than the sedimentation velocity of the particles [1]. Practically all of the studies regarding transport of solids in pipes have been developed using water as a carrier fluid. However, the rheological properties of the solids and water mixture can be modified depending on the concentration and mineralogy of the particles transported by the fluid. Thus, for high concentrations of solids, the particles with smaller size and the water behave as an equivalent non-Newtonian fluid which is the carrier of the larger particles. Studies considering non-Newtonian carriers are comparatively much less than those that considers water or a Newtonian transport fluid [2]. The objective of this article is to report the results of an experimental study regarding the concentration distribution of the solid particles transported by a pseudoplastic fluid in laminar regime and interpret them considering the diffusive fluxes due to gradient of concentration and gradient of viscosity.

\section{Experimental Set-up and Materials}

\subsection{Experimental set-up}

A sketch of the experimental set-up used in the research is shown in Fig. 1. From the head tank, the slurry is pumped towards the test section by means of two centrifugal pumps EBARA DWO400 connected in series and controlled by a frequency inverter SEW EURODRIVE model MOVITRAC B. The test section consisted in a $12 \mathrm{~m}$ long transparent PVC 
pipe with an inner diameter of $5.08 \mathrm{~cm}$. Along the pipe, 3 pressure transducers were installed to get continuous pressure records. Near the end of the pipe, an electrical resistance tomography (ERT) sensor with a data acquisition system model P2+ made by ITS was installed in order to record the concentration distribution of solids. The software used for data processing was ITS System 2000. The slurry returned to the head tank by means of a pipe of $2.54 \mathrm{~cm}$ diameter. A refrigerating system was installed along this pipe that kept the slurry at a constant temperature. Discharge was measured with a magnetic flowmeter SIEMENS 3100 and a signal transmitter SIEMENS MAG-500.

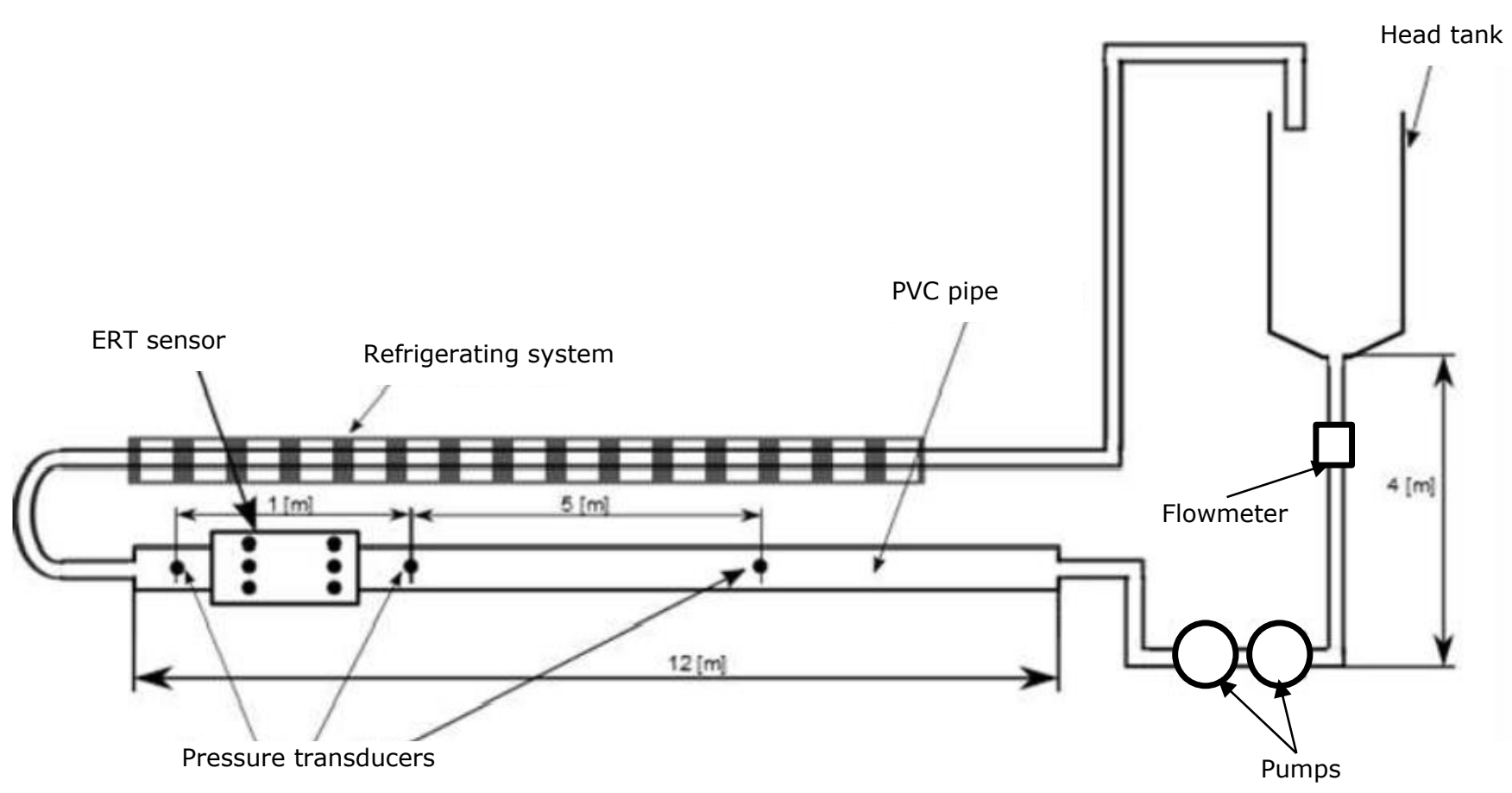

Fig. 1: Experimental set-up.

\subsection{Materials}

Glass microspheres of three different size distributions and density $(\rho)$ equal to $2500 \mathrm{~kg} / \mathrm{m}^{3}$ were the solid particles used in the experiments. Characteristic diameters of each size distribution are given in Table 1.

Table 1: Characteristic diameters of the glass microspheres used in the experiments.

\begin{tabular}{|c|c|c|c|}
\hline Type & $d_{20}(\mu \mathrm{m})$ & $d_{50}(\mu \mathrm{m})$ & $d_{80}(\mu \mathrm{m})$ \\
\hline I & 100 & 120 & 150 \\
\hline II & 205 & 300 & 380 \\
\hline III & 435 & 600 & 740 \\
\hline
\end{tabular}

The pseudoplastic fluid used as a carrier was generated dissolving sodium carboxymethyl cellulose (CMC) in water. When the solids particles are added to the fluid, the finest particles and the fluid behaves as an equivalent fluid and the rheology to this mixture needs to be known. Three different concentrations (weight/weight) of CMC in water were considered: $0.5 \%, 0.6 \%$ and $0.8 \%$, and concentrations (volume/volume) of solids in the CMC and water mixture ranging from nominal $17 \%$ to $32 \%$. Thus, a total of 26 different conditions associated to the materials were defined. The largest particles of the different types of solids present a process of sedimentation during the rheology determination, and it was found that particles with sizes smaller than 60-70 $\mu \mathrm{m}$ do not sediment through the rheological tests. The characteristic rheology corresponds to the mixture of fines particles and the pseudoplastic aqueous CMC solution. Rheology was determined with a rheometer made by Anton Paar model Rheolab QC, with a peltier temperature control. The rheograms fitted well to a Ostwald-de Waele (or power law) model, $\tau=K \dot{\gamma}^{n}$, where $\tau$ is the shear stress, $\dot{\gamma}$ is the strain rate, $K$ is the consistency coefficient and $n$ is the flow index. Density (fine particles+fluid) was measured with was measured by a Gamma RTM Dr J Ambrus densimeter. A summary of the parameter characterizing the mixtures and the solid concentrations $(\phi)$ are presented in Table 2. 


\subsection{Flow discharge}

The volumetric discharge $Q$ of the mixtures ranged between 0.030 and $1.748 \mathrm{~L} / \mathrm{s}$ depending on each specific the mixture, and it is given in Table 2 .

Table 2: Rheological parameters and concentration of solids in the experiments.

\begin{tabular}{|c|c|c|c|c|c|c|}
\hline Mixture No. & $d_{50}(\mu \mathrm{m})$ & $\phi(\%, \mathrm{vol} / \mathrm{vol})$ & $\rho\left(\mathrm{kg} / \mathrm{m}^{3}\right)$ & $K\left(\mathrm{~Pa} \cdot \mathrm{s}^{\mathrm{n}}\right)$ & $n$ & $Q(\mathrm{~L} / \mathrm{s})$ \\
\hline 1 & 120 & $19.278-27.221$ & 1005.025 & 0.29 & 0.69 & $0.538-1.636$ \\
\hline 2 & 120 & $16.767-23.411$ & 1005.025 & 0.13 & 0.83 & $0.470-1.396$ \\
\hline 3 & 120 & $18.292-28.648$ & 1005.025 & 0.44 & 0.66 & $0.302-1.258$ \\
\hline 4 & 120 & $21.813-25.524$ & 1006.899 & 0.81 & 0.61 & $0.323-0.996$ \\
\hline 5 & 120 & $21.979-31.643$ & 1006.899 & 1.12 & 0.58 & $0.108-0.968$ \\
\hline 6 & 120 & $23.709-31.078$ & 1006.899 & 1.31 & 0.54 & $0.085-0.762$ \\
\hline 7 & 120 & $17.355-20.595$ & 1008.065 & 2.94 & 0.46 & $0.197-1.149$ \\
\hline 8 & 120 & $22.718-24.273$ & 1008.065 & 3.34 & 0.47 & $0.083-1.020$ \\
\hline 9 & 300 & $18.330-41.107$ & 1005.025 & 0.36 & 0.66 & $0.069-1.748$ \\
\hline 10 & 300 & $24.385-47.449$ & 1005.025 & 0.43 & 0.64 & $0.050-1.584$ \\
\hline 11 & 300 & $29.368-44.152$ & 1005.025 & 0.73 & 0.58 & $0.099-1.677$ \\
\hline 12 & 300 & $19.614-41.622$ & 1006.899 & 0.81 & 0.56 & $0.050-1.287$ \\
\hline 13 & 300 & $23.841-43.549$ & 1009.899 & 0.65 & 0.59 & $0.114-1.735$ \\
\hline 14 & 300 & $27.680-43.138$ & 1006.899 & 0.92 & 0.57 & $0.091-1.497$ \\
\hline 15 & 300 & $17.008-32.324$ & 1008.065 & 3.63 & 0.45 & $0.030-0.473$ \\
\hline 16 & 300 & $24.551-41.744$ & 1008.065 & 3.26 & 0.44 & $0.076-1.338$ \\
\hline 17 & 300 & $30.508-46.760$ & 1008.065 & 3.52 & 0.42 & $0.030-1.162$ \\
\hline 18 & 600 & $22.251-38.988$ & 1005.025 & 0.22 & 0.71 & $0.069-1.748$ \\
\hline 19 & 600 & $26.137-41.421$ & 1005.025 & 0.21 & 0.71 & $0.159-1.584$ \\
\hline 20 & 600 & $29.928-44.486$ & 1005.025 & 0.14 & 0.77 & $0.099-1.677$ \\
\hline 21 & 600 & $20.897-33.029$ & 1006.899 & 0.31 & 0.68 & $0.358-1.400$ \\
\hline 22 & 600 & $27.317-42.342$ & 1006.899 & 0.32 & 0.67 & $0.237-1.197$ \\
\hline 23 & 600 & $28.687-41.318$ & 1006.899 & 0.23 & 0.71 & $0.231-1.340$ \\
\hline 24 & 600 & $25.000-43.511$ & 1008.065 & 1.14 & 0.55 & $0.030-0.819$ \\
\hline 25 & 600 & $26.600-43.387$ & 1008.065 & 0.77 & 0.59 & $0.107-1.132$ \\
\hline 26 & 600 & $32.189-52.278$ & 1008.065 & 0.38 & 0.68 & $0.139-1.233$ \\
\hline
\end{tabular}

\subsection{Flow regime}

To define the flow regime, the criterion by Mishra and Tripathi [3] was used. According to that criterion, the flow regime is laminar when the Reynolds number, as defined by Metzner and Reed [4], $R e=\frac{1}{8^{n-1}}\left(\frac{4 n}{3 n+1}\right)^{n} \frac{\rho U^{2-n} D^{n}}{K}$, is less than a critical value given by $R e_{C}=\frac{(4 n+2)(5 n+3)}{3(3 n+1)^{2}} 2100 . D$ and $U$ are the pipe diameter and flow velocity, respectively. For all the mixtures, the minimum value of $R e_{C}$ is 2186 and the maximum value of the Reynolds numbers for all the flow conditions is $R e=1287$, ensuring the laminar regime.

\section{Experimental Results}

A total of 241 concentration distributions of the solid particles (for equal number of flow conditions) were obtained with the electrical resistance tomography sensor. Grouped according to the size $d_{50}$ of the particles, the number of concentration distributions is: 78 for $d_{50}=120 \mu \mathrm{m}, 81$ for $d_{50}=300 \mu \mathrm{m}$ and 82 for $d_{50}=600 \mu \mathrm{m}$. Given the impossibility to show the measurements for all the experiments, 9 were chosen to be presented in this paper and they are shown in Fig. 2. (The complete set of measurements can be found in the appendix of the Master Thesis of the second author [5], downloadable from http://repositorio.uchile.cl/handle/2250/132978 ). Associated to the tomographic images presented in Fig. 2 are the 
concentration profiles along the vertical axis $z$ that are shown in Fig. 3. The value of the concentration for each location $z$ corresponds to the horizontal average.


Fig. 2: Concentration distributions obtained with the ETR sensor. Each column from left to right corresponds to $d_{50}=120 \mu \mathrm{m}, 300 \mu \mathrm{m}$ and $600 \mu \mathrm{m}$. Each file from bottom to top corresponds to the nominal discharges $Q=0.3 \mathrm{~L} / \mathrm{s}, 0.8 \mathrm{~L} / \mathrm{s}$ and $1.4 \mathrm{~L} / \mathrm{s}$. Concentration below $0 \%$ does not have physical sense. The value of $-10 \%$ was chosen to delimit the pipe boundaries.

The measured concentration presented unexpected distributions (at least for the authors), showing lower values near the centre of the pipe and sometimes with the highest values near the top of the pipe, as can be seen in Fig. 3. After reviewing the hardware, the data acquisition and data analysis protocols it was discarded that they were measurements artefacts or data misprocessing, and the results should be analysed considering the dynamics of a non-Newtonian fluid flow and its interaction with the solid particles.

\section{Analysis of experimental results}

The measurements show that the particles with $d_{50}$ equal to 120 and $300 \mu \mathrm{m}$ present a concentration distribution with two local maxima, one located in the upper half of the pipe and the second one in the lower half, with a region of low concentration in the centre of the conduit (under some conditions, the concentration at the centre of the pipe was zero). The distribution for the particles with $d_{50}=600 \mu \mathrm{m}$ follows a more common tendency, with higher values near the bottom, although lower values are still found near the centre of the pipe. Another important issue was that, although the solid particles are negatively buoyant, they are kept in suspension. Particles with $d_{50}=300$ and $600 \mu \mathrm{m}$ do not settle, even for flows with Reynolds numbers as low as $R e=100$.

The hypothesis that the concentration distribution of the solid particles and its suspension in laminar regime is the result of fluxes deriving from hydrodynamic diffusion process $[6,7]$ and the non-Newtonian characteristic of the fluid arose as an explanation. A qualitative analysis based on the diffusive model by Phillips et al. [8] was used to explain the shape of the concentration distribution of solids. It has to be noted that that model considers a Newtonian fluid with neutrally buoyant 
particles, and it has to be modified for an Ostwal-de Waele fluid and settling particles. According to Phillips et al model, the transport of concentration $\phi$ is given by:

$$
\frac{D \phi}{D t}=-\nabla \cdot\left(N_{C}+N_{\mu}\right)
$$

where $D \phi / D t$ is the material derivative of the concentration $\phi . N_{C}$ and $N_{\mu}$ are the fluxes of particles due to the gradient of concentration and to the gradient of viscosity, respectively, and they depend on dimensionless diffusion coefficients $D_{C}$ and $D_{\mu}$ that were determined experimentally by Phillips et al. The fluxes are given by:

$$
\begin{gathered}
N_{C}=-D_{C} a^{2} \phi \nabla(\dot{\gamma} \phi)=-D_{C} a^{2}\left(\phi^{2} \nabla \dot{\gamma}+\dot{\gamma} \phi \nabla \phi\right) \\
N_{\mu}=-D_{\mu} \dot{\gamma} \phi^{2}\left(\frac{a^{2}}{\mu_{m}}\right) \nabla \mu_{m}
\end{gathered}
$$

In the above equations, $a$ is the particle diameter and $\mu_{m}$ the viscosity of the mixture, which depends on the solids concentration. Note that the flux due to viscosity gradient cannot exist in the flow of a pure Newtonian fluid.



Fig. 3: Distribution of the horizontal averaged concentration. Each column from left to right corresponds to $d_{50}=120 \mu \mathrm{m}, 300 \mu \mathrm{m}$ and $600 \mu \mathrm{m}$. Each file from bottom to top corresponds to the nominal discharges $Q=0.3 \mathrm{~L} / \mathrm{s}, 0.8 \mathrm{~L} / \mathrm{s}$ and $1.4 \mathrm{~L} / \mathrm{s}$.

Phillips et al. model does not consider the downward flux resulting from the action of gravity. In the range of Stokes, this flux is given by

$$
N_{g}=-\frac{2}{9} f g \phi a^{2} \frac{\rho_{S}-\rho}{\mu_{e f f}}
$$

where $f$ is a hindering function:

$$
f=(1-\phi)^{\beta}
$$

with $\beta$ obtained from 


$$
\frac{4.8-\beta}{\beta-2.4}=0.0365\left(C_{D} \operatorname{Re}_{P}^{2 /(2-n)}\right)^{0.57}\left[1-2.4\left(\frac{d}{D}\right)^{0.27}\right]
$$

where $R e_{P}=\rho V^{2-n} a^{n} / K$ is the particle Reynolds number ( $V$ is the particle velocity) and $C_{D}$ the drag coefficient that can be computed from any available relationship, like that by Dhole et al. [9] which is valid in the range $5 \leq R e_{P} \leq 500$ : $C_{D}=\left(24 / \operatorname{Re}_{P}\right)\left(1+0.148 R e_{P}^{2.35 n /(2.42 n+0.918)}\right)$.

In order to carry out a qualitative analysis, the flow in a cylindrical pipe will be simplified to a two dimensional Pouseille flow, where the $Z$ direction is along the diameters of the pipe (Fig. 4). As gravity acts in the vertical direction, $N_{g}$ will be projected along $z$ in the analysis that follows. Assuming a steady state flow with no secondary currents, i.e. only with the component $u$ of the velocity in the $x$ direction, the momentum equation is reduced to $0=-\partial P / \partial x+\partial \tau_{z x} / \partial z$, where $z$ is the coordinate normal to $x, P$ is the pressure and $\tau_{z x}$ the shear stress that is reduced to $\tau_{z x}=$ $K(\partial u / \partial z)^{n}$. To simplify the notation, it is defined $P_{x}=\partial P / \partial x$ and $\dot{\gamma}=$ $\partial u / \partial z$. Integrating the momentum equation with respect to,$\dot{\gamma}=$



Fig. 4: Simplification of the cylindrical geometry to the two dimensional Poiseulille flow. $\left(\left(P_{x} z+C_{1}\right) / K\right)^{1 / n}$ is obtained, with $C_{1}$ a constant of integration. The mixture of fine particles and the psudoplastic carrier behave as an equivalent pseudoplastic fluid, characterized by a mixture consistency coefficient, $K_{m}$, which is a function of the volumetric concentration of solids, $\phi$. It can be estimated according to the relationships of Kawase and Ulbrecht (1983) [10]. Thus, the deformation shear rate of the mixture can be written as

$$
\dot{\gamma}_{m}=\left(\frac{P_{x} z+C_{1}}{K_{m}}\right)^{1 / n}
$$

An effective viscosity of the mixture is defined as

$$
\mu_{m}=K_{m}\left|\dot{\gamma}_{m}\right|^{n-1}=K_{m}\left|\frac{P_{x} z+C_{1}}{K_{m}}\right|^{\frac{n-1}{n}}
$$

Using $\dot{\gamma}_{m}$ instead of $\dot{\gamma}$, and $\mu_{m}$ in the expressions for the fluxes, and considering two dimensional Pouseuille flow, it is possible to get more manageable relationships that will allow us to know the flux of solid particles in the $2 \mathrm{D}$ pipe. Thus, the fluxes associated to concentration gradient and viscosity gradient are reduced to:

$$
\begin{gathered}
N_{C}=-D_{C} a^{2}\left(\phi^{2} \frac{d\left|\dot{\gamma}_{m}\right|}{d z}+\phi\left|\dot{\gamma}_{m}\right| \frac{d \phi}{d z}\right) \\
N_{\mu}=-D_{\mu}\left|\dot{\gamma}_{m}\right| \phi^{2}\left(\frac{a^{2}}{\mu_{m}}\right) \frac{d \mu_{m}}{d z} \\
\frac{d \mu_{m}}{d z}=\frac{d K_{m}}{d \phi} \frac{d \phi}{d z}
\end{gathered}
$$

With respect to the diffusion coefficients, $D_{C}=0.43$ [11] and $D_{\mu}=D_{C} /(0.01042 \phi+0.142)$ [12] were used. It is worth to stress the important role played by the energy loss $P_{x}$ through the mixture viscosity in all the fluxes. The absolute value of the shear rate should be used in the equations because the diffusive model is based on the frequency of particle collisions which scales with $\left|\dot{\gamma}_{m}\right|$, according to the model of Leighton and Acrivos [7,8].

It is easy to see that the direction of the fluxes $N_{C}$ and $N_{\mu}$ is defined by the sign of $d \phi / d z, d\left|\dot{\gamma}_{m}\right| / d z$ and $d \mu_{m} / d z$. The flux of particles due to gravity, $N_{g}$, depends only on $\left(\rho_{S}-\rho\right)$ and it is always downwards for negatively buoyant particles. The net flux of particles along the $z$ direction is $N_{T}=N_{C}+N_{\mu}+N_{g} \sin \theta$. The result of an analysis for different conditions is given below for some particular cases. 

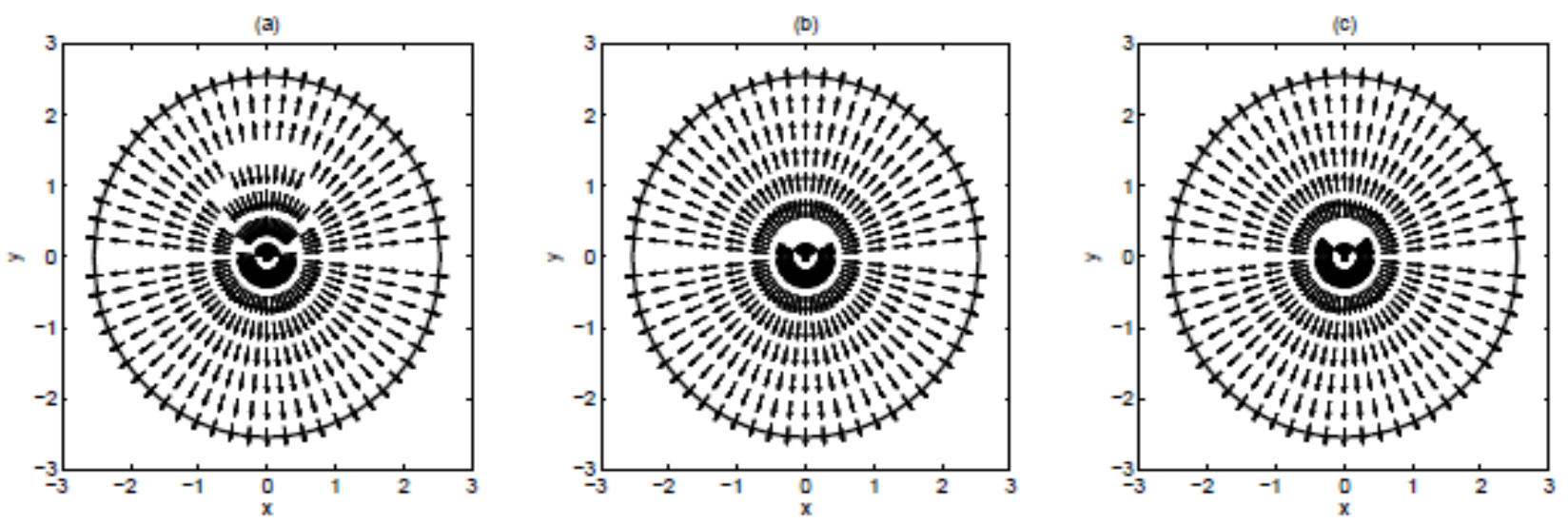

Fig. 5: Effect of viscosity in the direction of the flux of particles. $Q \sim 1 \mathrm{~L} / \mathrm{s}$ and $d_{50}=600 \mu \mathrm{m}$.

INCREASING PARTICLE SIZE
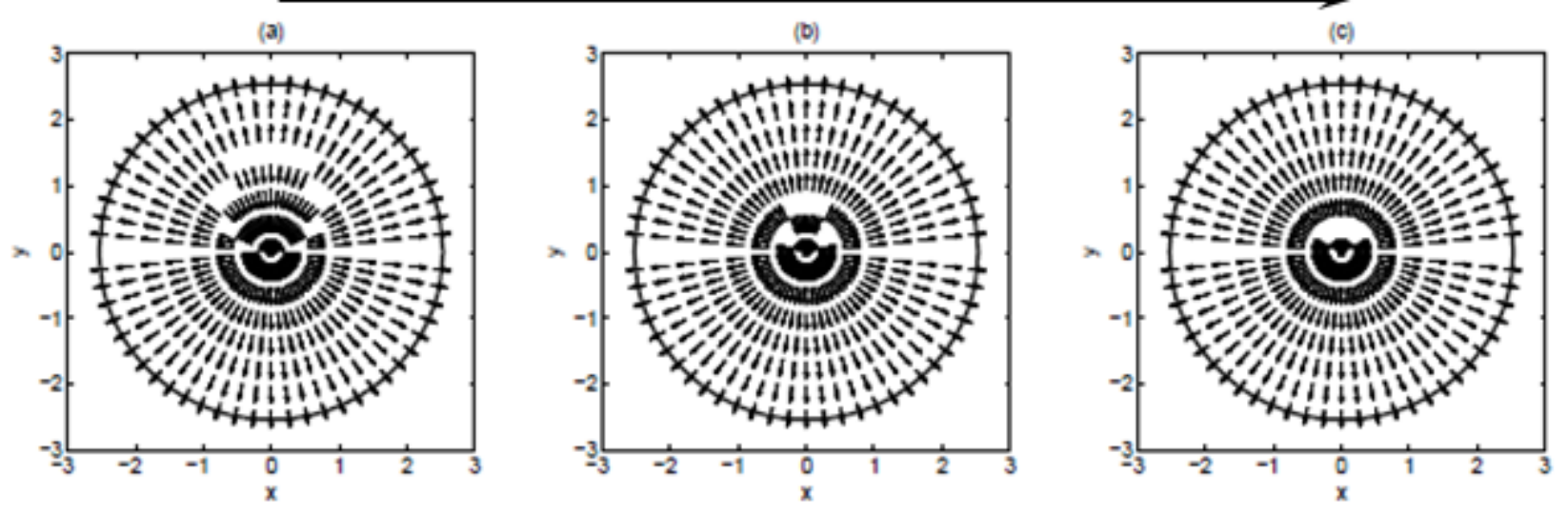

Fig. 6: Effect of particle size in the direction of the flux of particles. $Q \sim 1 \mathrm{~L} / \mathrm{s}$.
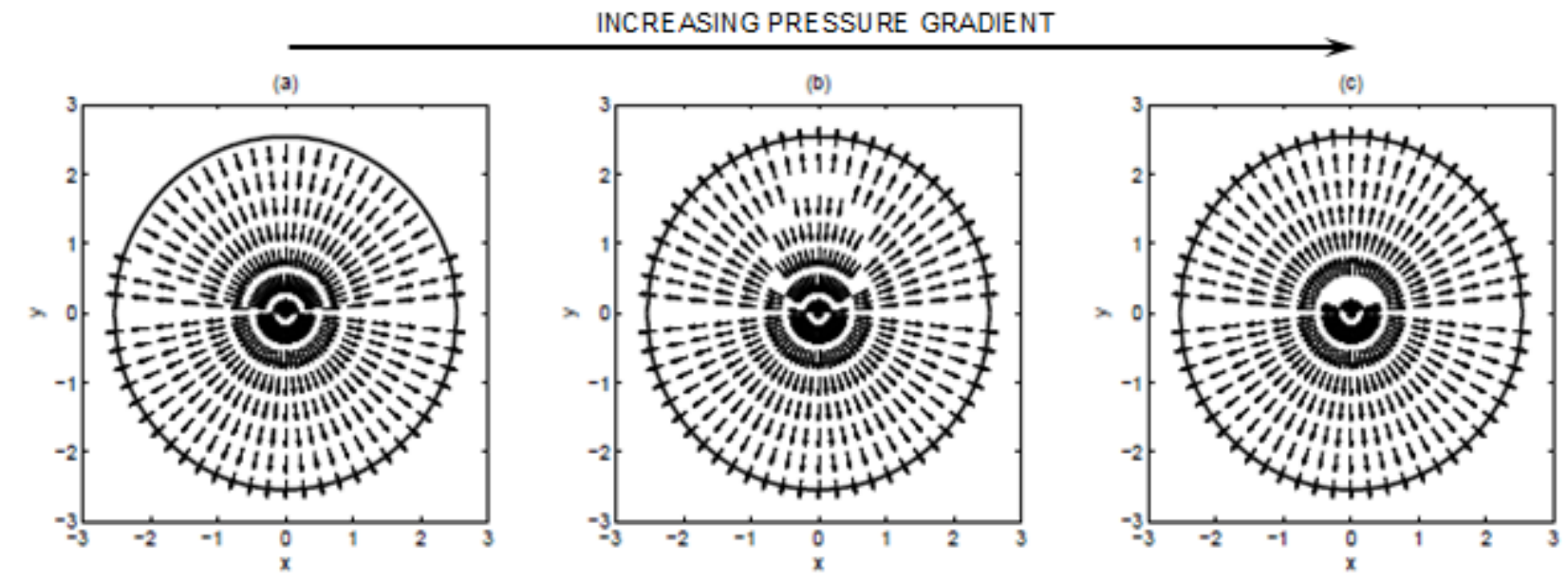

Fig. 7: Effect of the head loss in the direction of the fluxof particles. $Q \sim 1.6 \mathrm{~L} / \mathrm{s}$ and $d_{50}=120 \mu \mathrm{m}$.

For a relatively high discharge $(Q \sim 1 \mathrm{~L} / \mathrm{s})$ and $d_{50}=600 \mu \mathrm{m}$, it is found that $\left|N_{g} \sin \theta\right|>\left|N_{C}+N_{\mu}\right|$ only for the less viscous mixtures. Radial fluxes (along $z$ ) for this condition are presented in Fig. 5, in which viscosity increases from left to right. A similar analysis shows that for the same viscosity and discharge, $\left|N_{g} \sin \theta\right|>\left|N_{C}+N_{\mu}\right|$ in the centre of the pipe. Fig. 6 corresponds to the cases in which discharge, viscosity and pressure gradient are kept constant, changing the particle size. It is observed that for the two largest sizes of particles $\left(d_{50}\right.$ equal to 300 and $\left.600 \mu \mathrm{m}\right)$ gravity fluxes dominates only near the center of the pipe, with strong fluxes towards the walls due to the gradient of concentartion and viscosity. The effect 
of the head loss is presented in Fig. 7, where the flux directions are shown for $Q \sim 1.6 \mathrm{~L} / \mathrm{s}, d_{50}=600 \mu \mathrm{m}, K=0.29 \mathrm{~Pa} \cdot \mathrm{s}^{\mathrm{n}}$, and $n=0.60$, and three pressure gradients: $P_{x} / \rho_{m} g=0.10 \mathrm{~m}, 0.25 \mathrm{~m}, 0.30 \mathrm{~m}$. It is observed that at higher head loss per unit length, diffusive fluxes overcome the gravitational one.

\section{Conclusion}

The qualitative analysis of fluxes of solid particles due to gradient of concentration and viscosity indicates that the concentration of particles carried by a pseudoplastic fluid in laminar regime can present minimum values near the centre and higher close to the walls. The analysis, although highly simplified, preserves the most important physical mechanisms that govern the migration of the solid particles. Thus, it was explained why larger particles did not settle in the experiments. It was found that the pressure gradient (head loss) controls the fluxes through the effective viscosity of the mixture formed by the solid particles and the pseudoplasic fluid.

\section{Acknowledgements}

The authors acknowledge the funding provides by grants Fondecyt 1130910 and Fondequip EQM-120197. The second author also acknowledges the fellowship PFCHA/Magister Nacional/2013 folio 221320466.

\section{References}

[1] A. Oroskar and R. Turian, "The critical velocity in pipeline flow of slurries," AIChE Journal, vol. 26, no. 4, pp. 550$558,1980$.

[2] A. P. Poloski, F. Nigl, H. E. Adkins, M. J. Minette, J. Abrefah, J. J. Toth, A. M. Casella, J. M. Tingey, R. E. Hohimer, and S. T. Yokuda, "Deposition Velocities of Newtonian and Non-Newtonian Slurries in Pipelines," Pacific Northwest National Laboratory, PNNL-17639, WTP-RPT-175 Rev. 0, 2009.

[3] P. Mishra and G. Tripathi, "Transition from laminar to turbulent flow of purely viscous non-Newtonian fluids in tubes," Chemical Engineering Science, vol. 26, no. 6, pp. 915-921, 1971.

[4] A. Metzner and J. Reed, "Flow of non-Newtonian fluids-correlation of the laminar, transition, and turbulent-flow regions," AIChE Journal, vol. 1, no. 4, pp. 434-440, 1955.

[5] G. Montserrat, "Estudio experimental de las pérdidas de carga en tuberías con flujos de mezclas sólidos líquido nonewtoniano en régimen laminar," Master Thesis in Water Resources and Environment, Department of Civil Engineering, University of Chile, 2015.

[6] D. Leighton and A. Acrivos. "The shear-induced migration of particles in concentrated suspensions," Journal of Fluid Mechanics, vol. 181, pp. 415-439, 1987.

[7] D. Leighton and A. Acrivos, "Measurement of shear-induced self-diffusion in concentrated suspensions of spheres," Journal of Fluid Mechanics, vol. 177, pp. 109-131, 1987.

[8] R. Phillips, R. Brown, R., A. Graham, and J. Abbott. "A constitutive equation for concentrated suspensions that accounts for shear induced particle migration," Physics of Fluids, vol. 4, no. 1, pp. 30-40, 1992.

[9] S. D. Dhole, R. P. Chhabra, and V. Eswaran, "Flow of power-law fluids past a sphere at intermediate Reynolds numbers," Industrial and Engineering Chemistry Research, vol. 45, no. 13, pp. 4773-4781, 2006.

[10] Y. Kawase and J. J. Ulbrecht, "Rheological properties of suspensions of solid spheres in non-Newtonian fluids," Chemical Engineering Communications, vol. 20, no. 3-4, pp. 127-136, 1983.

[11] K. Zhang and A. Acrivos, "Viscous resuspension in fully developed laminar pipe flows," International journal of multiphase flow, vol. 20, no. 3, pp. 579-591, 1994.

[12] N. Tetlow, A. L. Graham, M. S. Ingber, S. R. Subia, L. A. Mondy, and S. A. Altobelli, "Particle migration in a Couette apparatus: Experiment and modeling," Journal of Rheology, vol. 42, no. 2, pp. 307-327, 1988. 